\title{
Metaanálisis de la frecuencia de JAK2 en mielofibrosis primaria según la técnica de detección
}

\author{
Meta-analysis of the frequency of JAK2 in primary \\ myelofibrosis according to the detection method \\ used
}

Mónica Mejía-Ochoa, Paola Andrea Acevedo-Toro, Jaiberth Antonio Cardona-Arias

- Medellín (Colombia)

DOI: https://doi.org/10.36104/amc.2020.1462

\section{Resumen}

Antecedentes y objetivo: en la literatura científica mundial la frecuencia de JAK2 presenta una alta heterogeneidad en las neoplasias mieloproliferativas crónicas. El objetivo de esta investigación fue analizar la prevalencia de la mutación JAK2 en mielofibrosis Primaria (MFP) y compararla según la técnica de detección en los años 2007-2018.

Material y métodos: revisión sistemática con metaanálisis, usando 21 búsquedas en tres bases de datos multidisciplinarias. Se aplicaron las fases de identificación, tamización, elección e inclusión de la guía PRISMA. Se garantizó reproducibilidad y evaluación de la calidad metodológica. Los análisis se basaron en frecuencias y metaanálisis para la prevalencia de la mutación con su intervalo de confianza de $95 \%$.

Resultados: se incluyeron 29 estudios con 744 pacientes, los cuales provienen principalmente de Corea, Brasil y China. La técnica más empleada fue AS-PCR, las prevalencias de JAK2 con esta técnica oscilaron entre 33.3 y $71.4 \%$; con PCR en tiempo real entre 42.9 y $77.3 \%$, con secuenciación de 14.3-57.4\% y en ARMS de 36.4-83.3\%. La prevalencia de JAK2 no presentó diferencias estadísticamente significativas según el tipo de prueba diagnóstica utilizada.

Conclusión: se evidencian altas frecuencias de la mutación JAK2V617F en MFP, lo que evidencia que el diagnóstico de esta entidad no debe realizarse únicamente por características clínicas y hematológicas sino también por la tamización genética de los pacientes. (Acta Med Colomb 2020; 45. DOI: https://doi.org/10.36104/amc.2020.1462).

Palabras clave: prevalencia; mutación; JAK2; mielofibrosis primaria; metaanálisis.

\footnotetext{
Abstract

Background and objective: in the global scientific literature, the frequency of JAK2 is highly heterogenous in chronic myeloproliferative neoplasms. The objective of this study was to analyze the prevalence of the JAK2 mutation in primary myelofibrosis (PMF) and compare it according to the detection method used, from 2007-2018.

Materials and methods: a systematic review with meta-analysis, using 21 searches in three multidisciplinary databases. The PRISMA guideline phases of identification, screening, selection and inclusion were applied. Reproducibility and evaluation of the methodological quality were ensured. The analyses were based on frequencies and meta-analysis for the prevalence of the mutation with its $95 \%$ confidence interval.

Results: twenty-nine studies with 744 patients were included, mainly from Korea, Brazil and China. The most commonly used technique was AS-PCR, and the prevalence of JAK2 with this technique ranged from 33.3 to $71.4 \%$; with real-time PCR ranging from 42.9 to $77.3 \%$, sequencing
}

Dra. Mónica Mejía-Ochoa: Bacterióloga, $\operatorname{MSc}(\mathrm{C})$ Microbiología y Bioanálisis-Énfasis Hematología. Grupo de Investigación Hematopatología Molecular, Escuela de Microbiología Universidad de Antioquia; Dra. Paola Andrea Acevedo-Toro: Microbióloga y Bioanalista, MSc Ciencias Básicas Biomédicas. Grupo de Investigación Hematopatología Molecular, Escuela de Microbiología Universidad de Antioquia; Dr. Jaiberth Antonio Cardona-Arias: MyB, MSc Epidemiología, MSc Economía Aplicada, $\mathrm{PhD}$ (candidato) Salud Pública. Escuela de Microbiología Universidad de Antioquia. Facultad de Medicina Universidad Cooperativa de Colombia. Medellín (Colombia).

Correspondencia: Dr. Jaiberth Antonio CardonaArias. Medellín (Colombia)

E-mail jaiberthcardona@gmail.com

Recibido: 30/VIII/2019 Aceptado: 07/X/2020 
from 14.3-57.4\%, and ARMS from 36.4-83.3\%. The prevalence of JAK2 showed no statistically significant differences according to the type of diagnostic test used.

Conclusion: high frequencies of the JAK2V617F mutation are seen in PMF, which shows that this entity should not be diagnosed solely based on clinical and hematological characteristics, but also on the patients' genetic screening. (Acta Med Colomb 2020; 45. DOI: https://doi.org/10.36104/ amc.2020.1462).

Key words: prevalence; mutation; JAK2; primary myelofibrosis; meta-analysis.

\section{Introducción}

La mielofibrosis primaria (MFP) es una neoplasia mieloproliferativa crónica (NMPC) filadelfia negativa; la más reciente clasificación de la Organización Mundial de la Salud la ha subclasificado en estado prefibrótico y fibrótico, dada la necesidad de diferenciarla de trombocitemia esencial. La incidencia anual estimada para esta enfermedad es 0.5-1.5 casos por 100000 personas, su prevalencia está en aumento debido al mejoramiento de su diagnóstico y la supervivencia $(1,2)$.

Al igual que las demás NMPC, la MFP se caracteriza por expansión clonal de células madres hematopoyéticas que conlleva a una producción descontrolada de células maduras, principalmente megacariocitos y granulocitos; una característica que diferencia esta entidad de las demás que componen el grupo es la fibrosis reactiva en médula ósea, con manifestaciones clínicas como anemia grave, esplenomegalia, trombosis y sangrado (3). Es importante mencionar que la fibrosis en médula ósea puede darse por causas diferentes a MFP, incluidos los estados de reactividad y entidades hematológicas como algunas leucemias agudas; en tales casos la mielofibrosis se denomina secundaria (4).

Un recurso diagnóstico que ha permitido diferenciar los tipos de mielofibrosis y esclarecer la patogénesis de las NMPC ha sido la detección de diferentes mutaciones (4), clasificadas en "drivers" y "otras mutaciones", éstas últimas relacionadas con pronóstico y evolución de la enfermedad. Las "mutaciones drivers" se utilizan como marcadores diagnósticos, para el caso de MFP se recomienda la detección de JAK2, CALR y MPL, siendo más relevante la primera $(1,4)$.

JAK2 es una proteína tipo Janus kinasa que participa en la vía de señalización JAK-STAT la cual regula diferentes procesos celulares como proliferación, diferenciación y apoptosis $(5,6)$. La alteración en JAK2 identificada en MFP es JAK2V617F, en la que una sustitución de aminoácidos genera un producto proteínico alterado responsable de la patogénesis de la enfermedad $(5,6)$.

Desde la identificación de JAK2 en MFP, el objetivo de varios estudios ha sido determinar su frecuencia, mostrando una elevada heterogeneidad, probablemente atribuible al tipo de población estudiada, así como a la variabilidad en los parámetros de validez diagnóstica de las pruebas empleadas para la detección del marcador. En tal sentido, se dispone de estudios con frecuencias tan bajas como de $14.3 \%$, reportada por Jaradat en 2015, y tan altas como de
80.0 y $83.3 \%$ reportadas por Suzuki en 2007 y Park en 2013, respectivamente (7-9).

Con base en estos antecedentes de investigación, el objetivo de esta revisión sistemática es metaanalizar la prevalencia de la mutación JAK2 en MFP y compararla según la técnica de detección.

\section{Material y métodos}

Tipo de estudio: revisión sistemática de la literatura con metaanálisis de medidas indirectas.

Protocolo de búsqueda y selección de estudios según la guía PRISMA Preferred reporting ítems for systematic reviews and meta-analyses (10)

Identificación: se realizó una búsqueda sin limitaciones temporales en las bases de datos multidisciplinarias Medline, Pubmed, Scielo y Science direct para la cual se emplearon los términos primary myelofibrosis, JAK2, Chromosome $P h$, Philadelphia chromosome, Philadelphia-Ph-chromosome, Philadelphia translocation y BCR ABL Negative. Cabe aclarar que la delimitación de la ventana de tiempo desde el 2007, se realizó a posteriori, con base en el estudio más antiguo hallado con el protocolo de revisión.

Tamización: se incluyeron los artículos que contenían los términos de búsqueda en título, resumen o palabras clave; y se eliminaron los títulos duplicados. Posteriormente se aplicaron como criterios de inclusión los estudios relacionados con el tema de interés (NMPC), investigaciones que reportaran la frecuencia de la mutación JAK2V617F en MFP, artículos originales y publicaciones en humanos o in vivo. Algunas sintaxis empleadas fueron: en Pubmed (((JAK2[Title) Abstract]) AND primary myelofibrosis[Title/Abstract]); chromosome ph[Title/Abstract]; BCR-ABL Negative[Titlel Abstract]; en Science-Direct TITLE-ABSTR-KEY(BCR ABL Negative) or TITLE-ABSTR-KEY(Chromosome Ph OR Philadelphia chromosome OR Ph chromosome OR Philadelphia translocation); TITLE-ABSTR-KEY(BCR ABL Negative), $y$ en Scielo (ti:((ab:(JAK2 primary myelofibrosis $))))$.

Elección: en la siguiente fase se excluyeron los artículos con un bajo número de pacientes (series de diez o menos casos), estudios con información incompleta que no especificaron tipo de diagnóstico o no reportaron la frecuencia de la mutación, estudios experimentales o clínicos y estudios que evaluaron pruebas diagnósticas.

Inclusión: la caracterización de los estudios se realizó con extracción de las variables título, autores, tipo de estudio, tema principal del estudio, revista, año de publicación, 
primer autor, país de estudio, número de pacientes evaluados, frecuencia de la mutación JAK2V617F, técnica de detección de la mutación y descripción de los sujetos de estudio.

Análisis de reproducibilidad y evaluación de la calidad metodológica: se evaluó la reproducibilidad de la búsqueda de estudios y extracción de la información con dos investigadores que aplicaron el protocolo de manera independiente, resolviendo las discrepancias por consenso. La calidad metodológica se determinó con la guía STROBE (Strengthening the Reporting of Observational studies in Epidemiology), cuyos criterios fueron aplicados por dos investigadores, con el fin de garantizar la reproducibilidad de esta fase.

\section{Análisis de la información}

Las variables de estudio se describieron con frecuencias absolutas y relativas, para el análisis de la frecuencia de la mutación JAK2V617F en MFP se realizó un metaanálisis de medidas indirectas según la técnica de detección (comparación de la prevalencia de la mutación según la técnica diagnóstica, pero a partir de estudios primarios que no hacen tal comparación, sino que reportaron la prevalencia de manera independiente por cada prueba de detección analizada), mediante la estimación de proporciones con su intervalo de confianza de $95 \%$ y Prueba Z (intervalos de confianza para la diferencia de proporciones).

\section{Resultados}

Se obtuvo un total de 12845 estudios sin la aplicación de límites, los cuales se restringen a 1909 resultados con la búsqueda en título, resumen, palabra clave; se eliminaron 253 artículos duplicados, 1482 artículos por no cumplir con los criterios de inclusión y 145 que cumplían con los criterios de exclusión, finalmente se tomaron 29 estudios para la síntesis cualitativa y cuantitativa de la información (Figura 1).

Los estudios se publicaron entre 2007 y 2018, los países con el mayor número de estudios fueron Corea $(n=5)$, Brasil $(n=3)$ y China $(n=3)$, el análisis por continente muestra que el mayor número de estudios provienen de Europa y Asia con $38 \%$ cada uno, seguido de América con $21 \%$ y finalmente África con 3\%. En los estudios que reportaron la edad promedio de los sujetos ésta fue mayor a 45 años, y en la mayoría se emplearon criterios diagnósticos de la OMS (Tabla 1).

Todos los estudios presentaron excelente calidad metodológica, dado que cumplieron con más de $70 \%$ de los criterios de la guía STROBE; sin embargo, la mayoría no explicitaron los parámetros para estimar el tamaño de muestra, ni discutió las limitaciones o posibilidades de generalización de los resultados (Figura 2).

Con base en la aplicación de AS-PCR las prevalencias de JAK2 oscilaron entre 33.3 y $71.4 \%$; con PCR en tiempo real el rango estuvo entre 42.9 y $77.3 \%$, con secuenciación de 14.3-57.4\% y en ARMS de 36.4-83.3\% (Figura 3). Dos estudios que utilizaron PCR-RFLP reportaron prevalencia

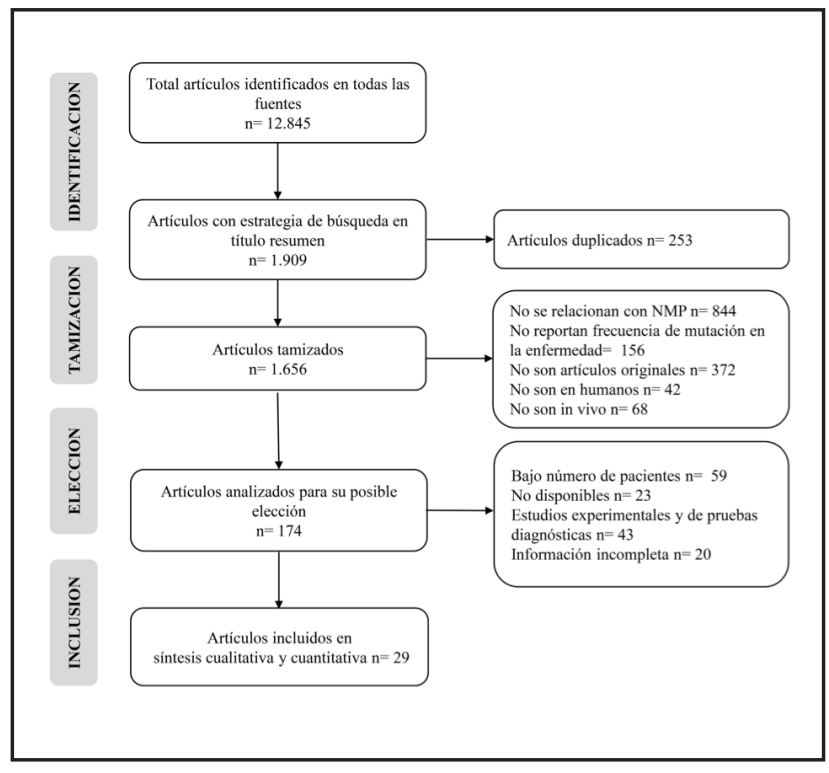

Figura 1. Flujograma de búsqueda y selección de estudios.

de 72.7 y $40 \%(32,32)$, en el estudio de Takata con SNP fue $36.4 \%$, Vytrva con DHPLC 63.6\%, Wu Z con HRM 58.0\% y Misawa con ABC-PCR 53.8\% (34-37).

\begin{tabular}{|c|c|}
\hline Criterios de la guía STROBE & Estudios que cumplen (\%) \\
\hline Título y resumen & 100 \\
\hline \multicolumn{2}{|l|}{ Introducción } \\
\hline Fundamentación & 100 \\
\hline Objetivo & 100 \\
\hline \multicolumn{2}{|l|}{ Métodos } \\
\hline Diseño estudio & 100 \\
\hline Descripción espacial - temporal & 100 \\
\hline Descripción de participantes & 100 \\
\hline Definición de variables & 100 \\
\hline Fuentes de datos y medición & 100 \\
\hline Control de sesgos & 90 \\
\hline Tamaño del estudio & 17 \\
\hline Variables Cuantitativas & 62 \\
\hline Métodos Estadísticos & 90 \\
\hline \multicolumn{2}{|l|}{ Resultados } \\
\hline Participantes & 100 \\
\hline Datos descriptivos & 100 \\
\hline Datos del desenlace central & 100 \\
\hline Resultados principales según el objetivo & 100 \\
\hline Análisis adicionales & 45 \\
\hline \multicolumn{2}{|l|}{ Discusión } \\
\hline Resultados clave & 97 \\
\hline Limitaciones & 34 \\
\hline Interpretación de resultados & 97 \\
\hline Generalización de resultados & 10 \\
\hline Financiacion del estudio & 62 \\
\hline
\end{tabular}

Figura 2. Evaluación de la calidad metodológica de los estudios. 
Mónica Mejía-Ochoa y cols.

Tabla 1. Descripción de estudios según año. país. edad y criterio diagnóstico.

\begin{tabular}{|c|c|c|c|c|}
\hline Autor & Año & País & Edad & Criterio diagnóstico \\
\hline \multicolumn{5}{|l|}{ AS-PCR } \\
\hline Speletas M (11) & 2007 & Grecia & $61.0^{\mathrm{a}}$ & Criterios Italianos para el diagnóstico \\
\hline Lucia E (12) & 2008 & Italia & Sin dato & OMS 2001 \\
\hline Pardanani A (13) & 2008 & Estados Unidos & $58.0^{\mathrm{a}}$ & OMS 2001 \\
\hline Xu W (14) & 2008 & China & $48.0^{\mathrm{b}}$ & OMS 2001 \\
\hline Bang S (15) & 2009 & Corea & Sin dato & OMS 2001 \\
\hline Medinger M (16) & 2009 & Suiza & $54.0^{\mathrm{a}}$ & No especifica \\
\hline Kim JT (17) & 2010 & Corea & $58.3^{\mathrm{b}}$ & OMS 2008 \\
\hline Benmoussa A (18) & 2011 & Marruecos & $56.83^{\text {a }}$ & No especifica \\
\hline Vadikolia CM (19) & 2011 & Grecia & $66.5^{\mathrm{a}}$ & OMS 2008 \\
\hline Ha J (20) & 2012 & Corea & $67.3^{\mathrm{a}}$ & OMS 2008 \\
\hline Zhang XY (21) & 2012 & China & Sin dato & No especifica \\
\hline \multicolumn{5}{|l|}{ PCR tiempo real } \\
\hline Boveri E (22) & 2008 & Italia & $58.0^{\mathrm{a}}$ & OMS 2001 \\
\hline Dos Santos L (23) & 2011 & Brasil & $59.3^{\mathrm{a}}$ & OMS 2008 \\
\hline Payzin KB (24) & 2014 & Turquía & $62.8^{\mathrm{a}}$ & OMS 2008 \\
\hline Azevedo AP (25) & 2017 & Portugal & Sin dato & OMS 2008 \\
\hline \multicolumn{5}{|l|}{ Secuenciación } \\
\hline Jaradat SA (7) & 2015 & Jordania & Sin dato a & No especifica \\
\hline $\operatorname{Kim} S(26)$ & 2015 & Corea & $61.5^{\mathrm{a}}$ & OMS 2008 \\
\hline Lekovic D (27) & 2017 & Serbia & $62.0^{\mathrm{a}}$ & OMS 2008 \\
\hline \multicolumn{5}{|l|}{ ARMS } \\
\hline Trifa AP (28) & 2010 & Rumania & $>60.0^{\mathrm{a}}$ & OMS 2001 \\
\hline Park SH (9) & 2013 & Corea & $62.0^{\mathrm{a}}$ & Expertos (histopatología) \\
\hline Borowczyk M (29) & 2015 & Polonia & $56.0^{\mathrm{a}}$ & OMS 2008 \\
\hline Ojeda MJ (30) & 2018 & Argentina & Sin dato & OMS 2008 \\
\hline \multicolumn{5}{|l|}{ PCR-RFLP } \\
\hline da Silva R (31) & 2012 & Brasil & Sin dato & Diagnóstico clínico \\
\hline Didone A (32) & 2016 & Brasil & $62.3^{\mathrm{b}}$ & OMS 2008 \\
\hline \multicolumn{5}{|l|}{ Otras } \\
\hline Tefferi A (RT-PCR) (33) & 2009 & Estados Unidos & $50.5^{\mathrm{a}}$ & OMS 2001 \\
\hline Takata Y (SNP) (34) & 2014 & Japón & $69.3^{\mathrm{a}}$ & OMS 2008 \\
\hline Vytrva N (DHPLC) (35) & 2014 & Austria & $72.9^{\mathrm{a}}$ & OMS 2001 \\
\hline Wu Z (HRM) (36) & 2014 & China & Sin dato & OMS 2008 \\
\hline Misawa K (ABC-PCR) (37) & 2018 & Japón & $60.0^{\mathrm{a}}$ & OMS 2008 \\
\hline
\end{tabular}


La prevalencia de JAK2 no presentó diferencias estadísticamente significativas según el tipo de prueba diagnóstica utilizada; con prevalencia de 64.6\% (IC95\%=54.7-74,6) usando ARMS en 99 pacientes; $57.3 \%$ (IC95\%=47.267.3) con PCR en tiempo real con 103 pacientes; $51.2 \%$ (IC95\%=44.1-58.3) en 205 pacientes evaluados con ASPCR y $51.0 \%$ (IC95\%=40.6-61.4) en 98 pacientes analiza-

\begin{tabular}{|c|c|c|}
\hline \multicolumn{3}{|l|}{ AS-PCR } \\
\hline Speletas M & 12 & 58,3 \\
\hline Lucia E & 19 & 63,2 \\
\hline Pardanani A & 37 & 51,4 \\
\hline Xu W & 13 & 38,5 \\
\hline Bang SM & 12 & 33,3 \\
\hline Medinger $\mathrm{M}$ & 26 & 53,8 \\
\hline Kim JT & 26 & 46,2 \\
\hline Benmoussa A & 12 & 33,3 \\
\hline Vadikolia CM & 14 & 71,4 \\
\hline Ha J & 17 & 52,9 \\
\hline Zhang XY & 17 & 52,9 \\
\hline \multicolumn{3}{|c|}{ PCR TIEMPO REAL } \\
\hline Boveri E & 46 & 56,5 \\
\hline Dos Santos L & 21 & 42,9 \\
\hline Payzin KB & 22 & 77,3 \\
\hline Azevedo AP & 14 & 50,0 \\
\hline \multicolumn{3}{|c|}{ SECUENCIACION } \\
\hline Jaradat SA & 14 & 14,3 \\
\hline $\operatorname{Kim~S}$ & 54 & 57,4 \\
\hline Lekovic D & 30 & 56,7 \\
\hline TOTAL & 98 & 51,0 \\
\hline \multicolumn{3}{|l|}{ ARMS } \\
\hline Trifa AP & 15 & 60,0 \\
\hline Park SH & 24 & 83,3 \\
\hline Borowczyk M & 11 & 36,4 \\
\hline Ojeda MJ & 49 & 63,3 \\
\hline
\end{tabular}

Figura 3. Prevalencia de JAK2 en los estudios que utilizaron PCR, secuenciación y ARMS.

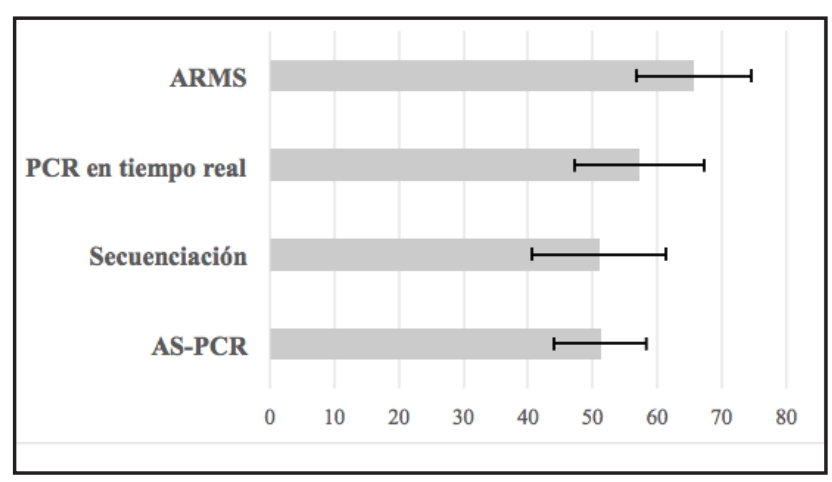

Figura 4. Metaanálisis de medidas indirectas para la prevalencia de JAK2 según el uso de PCR, secuenciación y ARMS. dos mediante secuenciación (Figura 4). No obstante, se debe aclarar que al comparar los grupos con la prevalencia más alta y la más baja, el poder estadístico fue $77.5 \%$, lo que daría cuenta de un error $\beta$ alto demostrando la necesidad de aumentar el número de investigaciones y de pacientes por estudio en esta enfermedad.

\section{Discusión}

Los resultados de esta revisión con 29 estudios y 744 pacientes evidencian que la principal técnica empleada fue AS-PCR, las prevalencias de JAK2 con esta técnica oscilaron entre 33.3 y $71.4 \%$; con PCR en tiempo real entre 42.9 y $77.3 \%$, con secuenciación de $14.3-57.4 \%$ y en ARMS de $36.4-83.3 \%$.

La mayoría de estudios se realizaron en Corea, Brasil y China, países con partidas presupuestales importantes en políticas de desarrollo e investigación (38). En el caso de Sur América, se evidencia un desarrollo insuficiente en la búsqueda de mutaciones en los diferentes centros hematológicos, a pesar de que JAK2 se incluya como criterio mayor de diagnóstico en la actualización de la OMS del año 2016; lo que impide el establecimiento de un diagnóstico preciso de la enfermedad que podría evitar la confusión con otras causas de fibrosis medular, al tiempo que impide un estudio integral de la enfermedad $(1,39)$.

Las altas frecuencias encontradas para la mutación JAK2 en este estudio ponen de manifiesto la necesidad de migrar de los sistemas de pronóstico convencionales basados principalmente en características clínicas como IPSS (Internacional Prognostic Scoring System) y DIPSS (Dinamyc Internacional Prognostic Scoring System), hacia el uso de nuevos sistemas que permiten la estratificación de riesgo de los pacientes basado en su perfil genético, principalmente con la detección de la mutación JAK2 (40-45).

En este orden de ideas, el bajo número de estudios en países latinoamericanos podría sugerir falta de adherencia a los criterios de puntuación pronóstica de los nuevos sistemas internacionales basados en características genéticas y moleculares como GIPSS (Genetically Inspired Prognostic Scoring System For Primary Myelofibrosis) y MIPSS (Mutation-Enhanced International Prognostic Scoring System) que permiten la estratificación precisa del riesgo de los pacientes, tanto al momento del diagnóstico como en el seguimiento de la enfermedad, en términos de supervivencia y riesgo de progresión a leucemia $(44,45)$.

Según la técnica utilizada, la prevalencia de las mutaciones en JAK2 no mostró una diferencia estadísticamente significativa, estos resultados pueden estar influenciados por un bajo poder estadístico $(77.5 \%)$ producto del bajo número de estudios y pacientes analizados por cada una de las técnicas; incluso, al evaluar la calidad metodológica sólo el 17\% describen cómo se llegó al tamaño de muestra, lo que afecta la estimación de la prevalencia. Esto puede estar sustentado en la dificultad para incluir pacientes con diagnóstico de MFP por su baja ocurrencia y búsqueda 
activa de casos a nivel mundial (46). Esta situación podría mejorarse con el desarrollo de estudios multicéntricos que permiten la inclusión de un mayor número de pacientes y de esta manera, mejorar la estimación de la prevalencia o aumentar el poder estadístico de las comparaciones realizadas en diferentes subgrupos.

El sistema de mutación refractaria a la amplificación (ARMS) y la PCR alelo específica (AS PCR) son métodos simples para detectar mutaciones que involucran cambios de una sola base o pequeñas delecciones, ambos pueden tener alta sensibilidad incluso con muy baja cantidad de células mutadas; sin embargo, este parámetro puede ser afectado por el tipo de mutación y diseño correcto de los primers (47). Por su parte, la PCR en tiempo real es altamente sensible y específica con tiempos de procesamiento muy cortos, a diferencia de las metodologías descritas, permite una cuantificación reproducible del material genético; no obstante, esta técnica puede generar gran cantidad de datos inexactos cuando no se tiene un estricto control de calidad de las variables analíticas como la calidad de los estándares y la correcta selección del gen housekeeping (48).

La secuenciación es una técnica molecular que permite determinar con exactitud modificaciones específicas en secuencias génicas; sin embargo, a diferencia de las anteriores, su principal limitación, cuando se usa secuenciación de Sanger, es la baja sensibilidad analítica, y las altas concentraciones de ADN requeridas (49).

En concordancia con estas características, y a pesar de que en este estudio no se hallaron diferencias entre las técnicas empleadas por el bajo poder estadístico de las comparaciones, es preciso recomendar el uso de metodologías de secuenciación diferentes a Sanger, con el fin de lograr mayor sensibilidad en la tamización genética de los pacientes con MFP.

Entre las limitaciones del presente estudio se encuentra el bajo tamaño de muestra de los estudios incluidos, lo que demuestra la necesidad de desarrollar nuevas investigaciones en el tema; además, por el número de estudios y pacientes en cada técnica no se desarrolló un metaanálisis con robustez en la evaluación estadística de la heterogeneidad, el sesgo de publicación o la sensibilidad de las medidas de resumen; esto podría guardar relación con las restricciones de idioma utilizados y las estrategias de búsqueda en cada fuente consultada. En este orden de ideas, estudios posteriores deberían mejorar la exhaustividad de selección de artículos en este campo mediante el aumento de los idiomas de búsqueda, ampliar el número de términos, de fuentes consultadas, entre otras estrategias que minimicen potenciales sesgos de selección.

Respecto a las técnicas empleadas en los diferentes estudios, la mayoría tienen alta sensibilidad; sin embargo, de acuerdo con las recomendaciones del grupo GEMFIN y la Sociedad Española de Hematología y Hemoterapia, no se recomienda el uso de metodologías como la secuenciación tipo Sanger, pirosecuenciación y PCR-RFLP por tener una sensibilidad baja que está limitada a un alto número de clonas mutadas (50).

\section{Conclusión}

Se evidenció una alta frecuencia de la mutación JAK2, demostrando que el diagnóstico de MFP no debe realizarse únicamente por características clínicas y hematológicas sino también por la búsqueda de marcadores moleculares específicos.

\section{Referencias}

1. Arber DA, Orazi A, Hasserjian R, Borowitz MJ, Beau MM Le, Bloomfield CD, et al. The 2016 revision to the World Health Organization classi fi cation of myeloid neoplasms and acute leukemia. Blood. 2016;127(20):2391-406.

2. SwerdlowS, Campo E, Harris $\mathbf{N}$ et al. World Health Organization Classification of Tumours of haematopoietic and lymphoid Tissues. 2017

3. Tefferi A. Primary myelofibrosis: 2017 update on diagnosis, risk-stratification, and management. Am J Hematol. 2016;91(12):1262-71.

4. Vainchenker W, Kralovics R. Genetic basis and molecular pathophysiology of classical myeloproliferative neoplasms. Blood. 2017;129(6):667-79.

5. Kralovics R, Passamonti F, Buser AS, Teo S-S, Tiedt R, Passweg JR, et al. A gain-of-function mutation of JAK2 in myeloproliferative disorders. $N$ Engl $J$ Med. 2005;352(17):1779-90.

6. Levine RL, Wadleigh M, Cools J, Ebert BL, Wernig G, Huntly BJP, et al. Activating mutation in the tyrosine kinase JAK2 in polycythemia vera, essential thrombocythemia, and myeloid metaplasia with myelofibrosis. Cancer Cell. 2005;7(4):387-97

7. Jaradat SA, Khasawneh R, Kamal N, Matalka I,Al-Bishtawi M,Al-Sweedan S, et al. Analysis of JAK2V617F mutation in Jordanian patients with myeloproliferative neoplasms. Hematol Oncol Stem Cell Ther. 2015;8(4):160-6.

8. Suzuki R, Onizuka M, Kojima M, Shimada M, Tsuboi K, Ogawa Y, et al. Infrequent hypermethylation of WIF-1 promoter in BCR/ABL-negative myeloproliferative disorders. Tokai J Exp Clin Med. 2007;32(4):131-5.

9. Park SH, Chi H-S, Cho Y-U, Jang S, Park C-J. The allele burden of JAK2 V617F can aid in differential diagnosis of Philadelphia Chromosome-Negative Myeloproliferative Neoplasm. Blood Res. 2013;48(2):128-32.

10. Moher D, Liberati A, Tetzlaff J, Altman DG, Group TP. Preferred Reporting Items for Systematic Reviews and Meta-Analyses: The PRISMA Statement. 2009;6(7).

11. Speletas M, Katodritou E, Daiou C, Mandala E, Papadakis E, Kioumi A, et al. Correlations of JAK2-V617F mutation with clinical and laboratory findings in patients with myeloproliferative disorders. Leuk Res. 2007;31(8):1053-62.

12. Lucia E, Martino B, Mammi C, Vigna E, Mazzone C, Gentile M, et al. The incidence of JAK2 V617F mutation in bcr/abl-negative chronic myeloproliferative disorders: assessment by two different detection methods. Leuk Lymphoma. 2008 ;4(10): 1907-15.

13. Pardanani A, Fridley BL, Lasho TL, Gilliland DG, Tefferi A. Host genetic variation contributes to phenotypic diversity in myeloproliferative disorders. Blood. 2008 ;111(5):2785-9.

14. Xu W, Li J-Y, Xia J, Zhang S-J, Fan L, Qiao C. MPL W515L mutation in Chinese patients with myeloproliferative diseases. Leuk Lymphoma. 2008;49(5):955-8.

15. Bang S-M, Lee J-S, Ahn JY, Lee JH, Hyun MS, Kim BS, et al. Vascular events in Korean patients with myeloproliferative neoplasms and their relationship to JAK2 mutation. Thromb Haemost. $2009 ; \mathbf{1 0 1 ( 3 ) : 5 4 7 - 5 1 . ~}$

16. Medinger M, Skoda R, Gratwohl A, Theocharides A, Buser A, Heim D, et al. Angiogenesis and vascular endothelial growth factor-/receptor expression in myeloproliferative neoplasms: correlation with clinical parameters and JAK2V617F mutational status. Br J Haematol. 2009;146(2):150-7.

17. Kim JT, Cho YG, Choi SI, Lee YJ, Kim HR, Jang SJ, et al. JAK2 V617F and exon 12 genetic variations in Korean patients with BCR/ABL1-negative myeloproliferative neoplasms. Korean J Lab Med. 2010;30(6):567-74.

18. Benmoussa A, Dehbi H, Fehri S, Quessar A, Nadifi S. JAK2-V617F mutation in Moroccan patients with myeloproliferative disorders: contribution, diagnosis and therapeutic prospects. Pathol Biol (Paris). 2011 ;59(4):e89-92.

19. Vadikolia CM, Tsatalas C, Anagnostopoulos K, Trypsianis G, Pantelidou D, Bazdiara I, et al. Proteolytic matrix metallopeptidases and inhibitors in BCRABL1-negative myeloproliferative neoplasms: correlation with JAK2 mutation status. Acta Haematol. 2011;126(1):54-62. 
20. Ha J-S, Kim Y-K, Jung S-I, Jung H-R, Chung I-S. Correlations between Janus kinase 2 V617F allele burdens and clinicohematologic parameters in myeloproliferative neoplasms. Ann Lab Med. 2012 ;32(6):385-91.

21. Zhang X, Maimaitili Y, Li Y, An L, Mao M, Fu L, et al. Detection and clinical significance of JAK2 V617F mutation in Chinese and Uyghur patients with chronic myeloproliferative in Xinjiang. Zhonghua Xue Ye Xue Za Zhi. 2012 ;33(12):1020-3.

22. Boveri E, Passamonti F, Rumi E, Pietra D, Elena C, Arcaini L, et al. Bone marrow microvessel density in chronic myeloproliferative disorders: a study of 115 patients with clinicopathological and molecular correlations. Br J Haematol. $2008 ; 140(2)$ : 162-8.

23. Dos Santos LC, Ribeiro JC da C, Silva NP, Cerutti J, da Silva MRR, Chauffaille M de LLF. Cytogenetics, JAK2 and MPL mutations in polycythemia vera, primary myelofibrosis and essential thrombocythemia. Rev Bras Hematol Hemoter. 2011;33(6):417-24.

24. Payzin KB, Savasoglu K, Alacacioglu I, Ozdemirkiran F, Mutlu BB, Bener S, et al. JAK2 V617F mutation status of 232 patients diagnosed with chronic myeloproliferative neoplasms. Clin Lymphoma Myeloma Leuk. 2014;14(6):525-33.

25. Azevedo AP, Silva SN, Reichert A, Lima F, Junior E, Rueff J. Prevalence of the Janus kinase 2 V617F mutation in Philadelphia-negative myeloproliferative neoplasms in a Portuguese population. Biomed reports. 2017;7(4):370-6.

26. Kim SY, Im K, Park SN, Kwon J, Kim J-A, Lee DS. CALR, JAK2, and MPL mutation profiles in patients with four different subtypes of myeloproliferative neoplasms: primary myelofibrosis, essential thrombocythemia, polycythemia vera, and myeloproliferative neoplasm, unclassifiable. Am J Clin Pathol. 2015 ;143(5):635-44

27. Lekovic D, Gotic M, Skoda R, Beleslin-Cokic B, Milic N, Mitrovic-Ajtic O, et al. Bone marrow microvessel density and plasma angiogenic factors in myeloproliferative neoplasms: clinicopathological and molecular correlations. Ann Hematol. 2017 ;96(3):393-404

28. Trifa AP, Cucuianu A, Petrov L, Urian L, Militaru MS, Dima D, et al. The G allele of the JAK2 rs 10974944 SNP, part of JAK2 46/1 haplotype, is strongly associated with JAK2 V617F-positive myeloproliferative neoplasms. Ann Hematol. $2010 ; \mathbf{8 9}(\mathbf{1 0})$ :979-83.

29. Borowczyk M, Wojtaszewska M, Lewandowski K, Gil L, Lewandowska M, Lehmann-Kopydlowska A, et al. The JAK2 V617F mutational status and allele burden may be related with the risk of venous thromboembolic events in patients with Philadelphia-negative myeloproliferative neoplasms. Thromb Res. 2015 ;135(2):272-80.

30. Ojeda MJ, Bragos IM, Calvo KL, Williams GM, Carbonell MM, Pratti AF. CALR, JAK2 and MPL mutation status in Argentinean patients with BCR-ABL1negative myeloproliferative neoplasms. Hematology. 2018;23(4):208-11.

31. da Silva RR, Domingues Hatzlhofer BL, Machado CG de F, Lima AS de M, de Albuquerque DM, dos Santos MNN, et al. JAK2 V617F mutation prevalence in myeloproliferative neoplasms in Pernambuco, Brazil. Genet Test Mol Biomarkers. $2012 ; \mathbf{1 6}(\mathbf{7}): 802-5$.

32. Didone A, Nardinelli L, Marchiani M, Ruiz ARL, de Lima Costa AL, Lima IS, et al. Comparative study of different methodologies to detect the JAK2 V617F mutation in chronic BCR-ABL1 negative myeloproliferative neoplasms. Pract Lab Med. 2016 ;4:30-7.

33. Tefferi A, Pardanani A, Lim K-H, Abdel-Wahab O, Lasho TL, Patel J, et al. TET2 mutations and their clinical correlates in polycythemia vera, essential thrombocythemia and myelofibrosis. Leukemia. 2009 ;23(5):905-11.

34. Takata Y, Seki R, Kanajii T, Nohara M, Koteda S, Kawaguchi K, et al. Association between thromboembolic events and the JAK2 V617F mutation in myeloproliferative neoplasms. Kurume Med J. 2014;60(3-4):89-97.
35. Vytrva N, Stacher E, Regitnig P, Zinke-Cerwenka W, Hojas S, Hubmann E, et al. Megakaryocytic morphology and clinical parameters in essential thrombocythemia, polycythemia vera, and primary myelofibrosis with and without JAK2 V617F. Arch Pathol Lab Med. 2014 ;138(9):1203-9.

36. Wu Z, Zhang X, Xu X, Chen Y, Hu T, Kang Z, et al. The mutation profile of JAK2 and CALR in Chinese Han patients with Philadelphia chromosome-negative myeloproliferative neoplasms. J Hematol Oncol. 2014 ; 7:48.

37. Misawa K, Yasuda H, Araki M, Ochiai T, Morishita S, Shirane S, et al. Mutational subtypes of JAK2 and CALR correlate with different clinical features in Japanese patients with myeloproliferative neoplasms. Int J Hematol. 2018 ;107(6):673-80.

38. . Grupo Banco mundial: Gasto en investigación y desarrollo (\% del PIB) [internet] n.d [consultado 10 de julio 2018]. Disponible en:https://datos.bancomundial.org/ indicador/GB.XPD.RSDV.GD.ZS?view=map\&year_high_desc=false.

39. Abello V, Quintero G, Espinosa D, Solano M, Casas C, D Saavedra. Descripción de las características clínicas de las neoplasias mieloproliferativas crónicas ( NMPC) Description of the clinical characteristics of chronic myeloproliferative neoplasms ( MPNs ) First report of the colombian registry of MPNs. 2017;35-41.

40. Passamonti F, Cervantes F, Vannucchi AM, Morra E, Rumi E, Cazzola M, et al. Dynamic International Prognostic Scoring System (DIPSS) predicts progression to acute myeloid leukemia in primary myelofibrosis. Vol. 116, Blood. United States; 2010. p. 2857-8.

41. Gangat N, Caramazza D, Vaidya R, George G, Begna K, Schwager S, et al. DIPSS plus: a refined Dynamic International Prognostic Scoring System for primary myelofibrosis that incorporates prognostic information from karyotype, platelet count, and transfusion status. J Clin Oncol. 2011 ;29(4):392-7.

42. Cervantes F, Dupriez B, Pereira A, Passamonti F, Reilly JT, Morra E, et al. New prognostic scoring system for primary myelofibrosis based on a study of the International Working Group for Myelofibrosis Research and Treatment. Blood. 2009 ;113(13):2895-901.

43. Mesa RA, Passamonti F. Individualizing Care for Patients With Myeloproliferative Neoplasms: Integrating Genetics, Evolving Therapies, and Patient-Specific Disease Burden. Am Soc Clin Oncol Educ book Am Soc Clin Oncol Annu Meet. 2016;35:e324-35.

44. Tefferi A, Guglielmelli P, Nicolosi M, Mannelli F, Mudireddy M, Bartalucci $\mathbf{N}$, et al. GIPSS: genetically inspired prognostic scoring system for primary myelofibrosis. Leukemia [Internet]. 2018;32(7):1631-42.

45. Tefferi A, Guglielmelli P, Lasho TL, Gangat N, Ketterling RP, Pardanani A, et al. MIPSS70+ Version 2.0: Mutation and Karyotype-Enhanced International Prognostic Scoring System for Primary Myelofibrosis. J Clin Oncol. 2018 ;36(17):1769-70.

46. Titmarsh GJ, Duncombe AS, Mcmullin MF, O'Rorke M, Mesa R, De Vocht $\mathbf{F}$, et al. How common are myeloproliferative neoplasms? A systematic review and meta-analysis. Am J Hematol. 2014;89(6):581-7.

47. Little S. Amplification-refractory mutation system (ARMS) analysis of point mutations. Curr Protoc Hum Genet. 2001; Chapter 9:Unit 9.8.

48. Arya M, Shergill IS, Williamson M, Gommersall L, Arya N, Patel HRH. Basic principles of real-time quantitative PCR. Expert Rev Mol Diagn. 2005 Mar;5(2):209-19.

49. Tipu HN, Shabbir A. Evolution of DNA sequencing. J Coll Physicians Surg Pak. $2015 ; 25(3): 210-5$.

50. Blessés C, Cervantes F. Manual de recomendaciones en Neoplasias Mieloproliferativas Crónicas Filadelfia Negativas. Grupo Español de Neoplasias Filadelfia Negativas (GEMFIN). 2014; Available from: http://www.sehh.es/images/stories/ recursos/2014/documentos/guias/GUIA_GEMFIN.pdf 